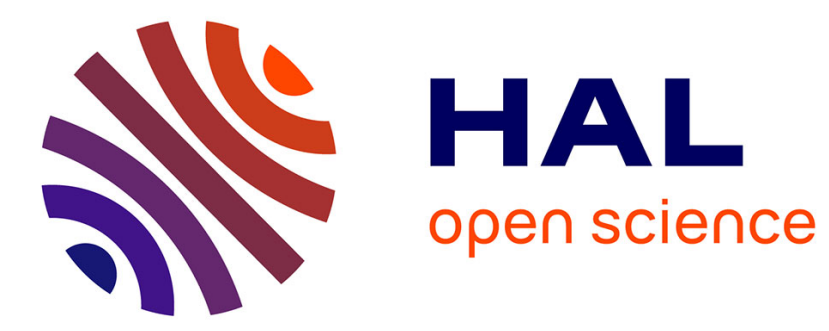

\title{
On Inverse Kinematics with Nonlinear Criteria: Trajectory Relaxation
}

Kévin Dufour, Wael Suleiman

\section{To cite this version:}

Kévin Dufour, Wael Suleiman. On Inverse Kinematics with Nonlinear Criteria: Trajectory Relaxation. 15th International Workshop on Advanced Motion Control (AMC), Mar 2018, Tokyo, Japan. 10.1109/AMC.2019.8371070 . hal-01700526

\section{HAL Id: hal-01700526 \\ https://hal.science/hal-01700526}

Submitted on 4 Feb 2018

HAL is a multi-disciplinary open access archive for the deposit and dissemination of scientific research documents, whether they are published or not. The documents may come from teaching and research institutions in France or abroad, or from public or private research centers.
L'archive ouverte pluridisciplinaire HAL, est destinée au dépôt et à la diffusion de documents scientifiques de niveau recherche, publiés ou non, émanant des établissements d'enseignement et de recherche français ou étrangers, des laboratoires publics ou privés. 


\title{
On Inverse Kinematics with Nonlinear Criteria: Trajectory Relaxation
}

\author{
Kévin Dufour and Wael Suleiman \\ Electrical and Computer Engineering Department, Faculty of Engineering \\ University of Sherbrooke, Canada \\ \{Kevin.K.Dufour, Wael.Suleiman\}@USherbrooke.ca
}

\begin{abstract}
The concept of trajectory relaxation of Inverse Kinematics (IK) problem is thoroughly investigated in this paper. Trajectory relaxation refers to relaxing the hard-constraint of following a desired trajectory, hence the robot is allowed to deviate from that trajectory. The main advantages of this concept is improving the robustness of the IK solver and efficiently allowing the optimization of additional nonlinear criteria, for instance maximizing the manipulability index of the robot.

We propose different formulations of the trajectory relaxation as well as several functions to control the stiffness of the relaxed constraint. We conducted experiments in simulation to compare the proposed formulations and identify their strengths and weaknesses, we also validated the results on a Baxter research robot.
\end{abstract}

\section{INTRODUCTION}

Inverse Kinematics is a classical problem in robotics addressing the generation of the command of a robot in order to execute a task. It usually calculates the joint variables to reach a Cartesian pose, it can be solved analytically or numerically. The latter is more popular as it gives a more generalized formulation into which it is easier to integrate several constraints or objectives. In this paper, we use this method to make a collaborative robot, Baxter from Rethink Robotics, follow a trajectory within the physical limitation of its joints. We consider minimizing the joint velocity norm subject to the trajectory constraints and velocity limits, it is also possible to add other objectives to be minimized or maximized.

In previous work [1], we studied the maximization of the manipulability index by formulating the IK problem as a particular case of an optimization problem, which is the Quadratic Programming (QP) problem. An important feature that we used is the trajectory relaxation, it allows the robot to deviate from its trajectory, hence giving more liberty to optimize the objectives more efficiently or to satisfy the constraints; in particular it is useful to avoid an obstacle that is on the way of the trajectory and thus to solve problems that would be unfeasible otherwise. However, to ensure the feasibility of the problem at the beginning of the movement, there should be a transition in the constraint of the trajectory so the deviation increases smoothly. Such a transition should also exist at the end of the trajectory so the robot effectively reaches the desired pose at the end of the task. The design of this transition is a challenging task as its formulation should ensure the smoothness and continuity of the movement and prevent major joint velocity oscillations or significant
Cartesian velocities. In this paper, this issue is extensively addressed by studying several transition functions.

The main contributions of the paper are:

- Proposing new formulations of the transition function or improving some existing ones in Section III-B.

- Comparing the impact of those functions on the movement of the robot and giving some insight into the methodology to determine the correct parameters. Tests have been conducted in simulation and validated with a real robot, the results are given in Section IV and are obtained by following a trajectory while maximizing the manipulability index, so that the robot deviates from the trajectory.

\section{InVERse Kinematics Problem Formulation}

The general formulation of the IK problem to follow a trajectory while maximizing or minimizing an additional criterion can be formulated as follows:

$$
\begin{array}{cl}
\underset{\dot{\boldsymbol{q}}, \boldsymbol{q}}{\operatorname{minimize}} & \frac{1}{2} \dot{\boldsymbol{q}}^{T} Q \dot{\boldsymbol{q}} \pm \alpha f(\boldsymbol{q}) \\
\text { subject to } & J \dot{\boldsymbol{q}}=\dot{\boldsymbol{r}} \\
& A \dot{\boldsymbol{q}} \leq \boldsymbol{b} \\
& \hat{\dot{\boldsymbol{q}}}^{-} \leq \dot{\boldsymbol{q}} \leq \hat{\dot{\boldsymbol{q}}}^{+}
\end{array}
$$

with $\boldsymbol{q}, \dot{\boldsymbol{q}} \in \mathbb{R}^{n}$ are respectively the joint variable and velocity, $Q$ is a diagonal and positive semi-definite weighting matrix, $f(\boldsymbol{q})$ is the additional objective to be maximized or minimized, it is in general nonlinear and non-convex function, and $\alpha$ is a positive coefficient. $\mathbf{J}$ is the Jacobian matrix of the robot end-effector and $\dot{r}$ is the velocity of the operational space trajectory. $A$ and $\boldsymbol{b}$ represent the constraints that result from considering geometric constraints, such as collision avoidance, or avoiding the obstruction of the robot visual field, for more details the reader is referred to [2], [3], [4], [5], [6]. Finally, $\hat{\dot{q}}^{+}$and $\hat{\dot{q}}^{-}$are the bounds of $\dot{\boldsymbol{q}}$ defined by the velocity damper formula [7].

An example of the additional criterion is maximizing the manipulability index. In this case the optimization problem (1) becomes:

$$
\begin{array}{cl}
\underset{\dot{\boldsymbol{q}}, \boldsymbol{q}}{\operatorname{minimize}} & \frac{1}{2} \dot{\boldsymbol{q}}^{T} Q \dot{\boldsymbol{q}}-\alpha m(\boldsymbol{q}) \\
\text { subject to } & J \dot{\boldsymbol{q}}=\dot{\boldsymbol{r}} \\
& A \dot{\boldsymbol{q}} \leq \boldsymbol{b} \\
& \hat{\dot{\boldsymbol{q}}}^{-} \leq \dot{\boldsymbol{q}} \leq \hat{\dot{\boldsymbol{q}}}^{+}
\end{array}
$$


where $m(\boldsymbol{q})=\sqrt{\operatorname{det}\left(J J^{T}\right)}$ is the manipulability index, measuring the closeness of a configuration to singularity [8], the value of $m(\boldsymbol{q})$ tends to zero when reaching a singular configuration. As discussed in [1], the optimization problem (2) is a nonlinear optimization problem which is hard to solve within a fixed time and therefore the hard real time constraint might not be satisfied.

However, as the optimization problem (1) is solved sequentially over a time interval $\mathrm{T}$, which is robot's control sampling period, the function $f(\boldsymbol{q})$ can be linearized as follows:

$$
\begin{aligned}
f\left(\boldsymbol{q}_{\boldsymbol{t}}\right) & =f\left(\boldsymbol{q}_{\boldsymbol{t}-\mathbf{1}}\right)+(\nabla f)^{T} \Delta \boldsymbol{q} \\
& =f\left(\boldsymbol{q}_{\boldsymbol{t}-\mathbf{1}}\right)+T(\nabla f)^{T} \dot{\boldsymbol{q}}
\end{aligned}
$$

where $\nabla f$ is the gradient of the function $f$ with respect to the joint variable $\boldsymbol{q}$. As a result the problem (1) is transformed into the following equivalent problem:

$$
\begin{array}{cl}
\underset{\dot{\boldsymbol{q}}}{\operatorname{minimize}} & \frac{1}{2} \dot{\boldsymbol{q}}^{T} Q \dot{\boldsymbol{q}} \pm \alpha T(\nabla f)^{T} \dot{\boldsymbol{q}} \\
\text { subject to } & J \dot{\boldsymbol{q}}=\dot{\boldsymbol{r}} \\
& A \dot{\boldsymbol{q}} \leq \boldsymbol{b} \\
& \hat{\boldsymbol{q}}^{-} \leq \dot{\boldsymbol{q}} \leq \hat{\dot{\boldsymbol{q}}}^{+}
\end{array}
$$

The above optimization problem has the form of a standard QP problem:

$$
\begin{array}{cl}
\underset{\boldsymbol{x}}{\operatorname{minimize}} & \frac{1}{2} \boldsymbol{x}^{T} Q \boldsymbol{x}+\boldsymbol{g}^{T} \boldsymbol{x} \\
\text { subject to } & C \boldsymbol{x}=\boldsymbol{e} \\
& \boldsymbol{b}^{-} \leq A \boldsymbol{x} \leq \boldsymbol{b}^{+} \\
& \hat{\boldsymbol{x}}^{-} \leq \boldsymbol{x} \leq \hat{\boldsymbol{x}}^{+}
\end{array}
$$

Even though the optimization problem has been transformed into a QP problem that can be efficiently solved in real time using an appropriate QP solver, such as qpOASES solver [9], the robot can only use redundancy in order to satisfy the kinematic task and maximize or minimize the additional criterion.

\section{TRAJECTORY RELAXATION}

The task of following the trajectory in (4) is expressed as a hard equality constraint. However, in some cases the problem can become unsolvable, for example when the trajectory passes through an unforeseen obstacle. Moreover if there are several objectives to optimize and the robot has very few redundancy, then it would not be possible to significantly improve the objectives while satisfying that constraint. A solution to that problem is to allow the robot to deviate from its trajectory during a period of time, which we call hereafter trajectory relaxation. In Section III-A, we present two formulations for the trajectory relaxation, and in Section III-B the transition between the hard and relaxed constraint states is thoroughly studied.

\section{A. Definition}

The relaxation of the trajectory has been defined in [10] by introducing a slack variable $\delta$ into the QP problem (4):

$$
\begin{array}{cl}
\underset{\dot{\boldsymbol{q}}, \boldsymbol{\delta}}{\operatorname{minimize}} & \frac{1}{2} \dot{\boldsymbol{q}}^{T} Q \dot{\boldsymbol{q}} \pm \alpha T(\nabla f)^{T} \dot{\boldsymbol{q}}+\frac{1}{2} \boldsymbol{\delta}^{T} Q_{\delta} \boldsymbol{\delta} \\
\text { subject to } & J \dot{\boldsymbol{q}}+\boldsymbol{\delta}=\dot{\boldsymbol{r}} \\
& A \dot{\boldsymbol{q}} \leq \boldsymbol{b} \\
& \hat{\dot{\boldsymbol{q}}}^{-} \leq \dot{\boldsymbol{q}} \leq \hat{\boldsymbol{q}}^{+} \\
& \boldsymbol{\delta}^{-} \leq \boldsymbol{\delta} \leq \boldsymbol{\delta}^{+}
\end{array}
$$

with $\delta \in \mathbb{R}^{p}$ having the same dimension as $\dot{\boldsymbol{r}}$. The norm of $\boldsymbol{\delta}$ is being minimized by the objective function and the stiffness of this objective is controlled by the weighting matrix $Q_{\delta}$, with $Q_{\delta}(t)=f_{e}(t) I_{p}, I_{p} \in \mathbb{R}^{p \times p}$ is the identity matrix and $f_{e}$ is a positive function ensuring the continuous variation of the stiffness, that will be further discussed in III-B.

In this formulation, the equality constraint becomes $J \dot{\boldsymbol{q}}+$ $\boldsymbol{\delta}=\dot{\boldsymbol{r}}$, meaning the robot follows the modified trajectory $\dot{\boldsymbol{r}}-\boldsymbol{\delta}$ and that $\boldsymbol{\delta}$ is the deviation in the velocity domain from the reference trajectory. The equality constraint can also be changed for an even less hard constraint:

$$
-\delta \leq J \dot{q}-\dot{\boldsymbol{r}} \leq \boldsymbol{\delta}
$$

As the objective function of a QP problem has a quadratic term, $\frac{1}{2} \boldsymbol{x}^{T} Q \boldsymbol{x}$ in (5), and a linear term, $\boldsymbol{g}^{T} \boldsymbol{x}$ in (5), we introduce a new formulation, adding a linear part relative to the slack variable:

$$
\begin{array}{cl}
\underset{\dot{\boldsymbol{q}}, \boldsymbol{\delta}}{\operatorname{minimize}} & \frac{1}{2} \dot{\boldsymbol{q}}^{T} Q \dot{\boldsymbol{q}} \pm \alpha T(\nabla f)^{T} \dot{\boldsymbol{q}}+\frac{1}{2} \boldsymbol{\delta}^{T} Q_{\delta} \boldsymbol{\delta}+\boldsymbol{\xi}^{T} \boldsymbol{\delta} \\
\text { subject to } & -\boldsymbol{\delta} \leq J \dot{\boldsymbol{q}}-\dot{\boldsymbol{r}} \leq \boldsymbol{\delta} \\
& A \dot{\boldsymbol{q}} \leq \boldsymbol{b} \\
& \hat{\boldsymbol{q}}^{-} \leq \dot{\boldsymbol{q}} \leq \hat{\boldsymbol{q}}^{+} \\
& \mathbf{0} \leq \boldsymbol{\delta} \leq \boldsymbol{\delta}^{+}
\end{array}
$$

with $\boldsymbol{\xi}(t)=f_{e}(t) \mathbf{1}$. Moreover, $\boldsymbol{\delta}$ must be positive so that minimizing $\boldsymbol{\xi}^{T} \boldsymbol{\delta}$ leads to minimizing the norm of $\boldsymbol{\delta}$.

\section{B. Transition Profiles}

The amplitude of the deviation is controlled by the bounds $\delta^{+}$and $\delta^{-}$of the slack variable and its associated transition function $f_{e}$, which is defined as follows:

$$
f_{e}(t)= \begin{cases}f_{1}(t) & \text { if } 0 \leq t \leq T_{\rho} \\ Q_{l o w} & \text { if } T_{\rho} \leq t \leq T_{f}-T_{\rho} \\ f_{2}(t) & \text { otherwise }\end{cases}
$$

with $f_{1}$ and $f_{2}$ functions defined to obtain a profile similar to Fig. 1 in order to have a smooth, continuous behavior for $\delta$, and as a result for $\dot{\boldsymbol{q}}$. The transition function, $f_{e}$, varies between two extreme values, which are $Q_{h i g h}$ at the beginning and at the end of the trajectory, so the movement is continuous from the start pose and recovers to the desired goal at the end, and $Q_{\text {low }}$ during the relaxed period. Thus, $Q_{\text {high }}$ should be high enough so the stiffness constraint of minimizing the norm of $\boldsymbol{\delta}$ is hard and the deviation is close 
to zero, and $Q_{\text {low }}$ should be low enough so that the constraint is soft and allows the deviation.

Thereafter, different formulations of $f_{e}$ will be extensively studied.

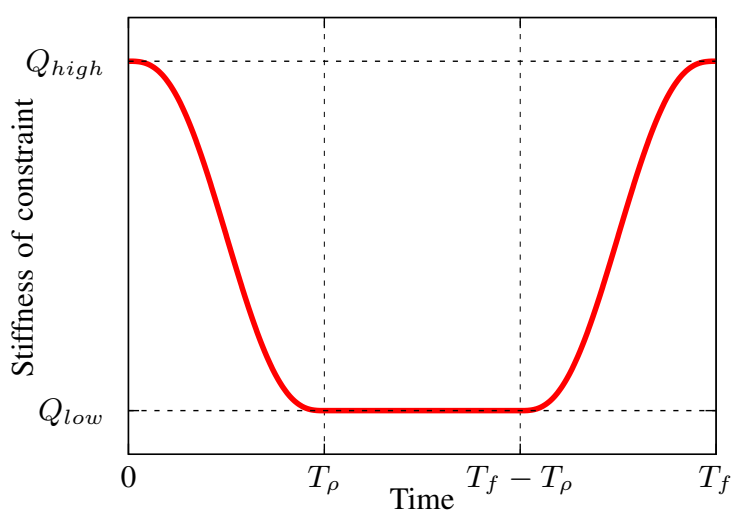

Fig. 1. Example of constraint stiffness profile ( $5^{\text {th }}$ order)

1) Hyperbolic Tangent Transition: The hyperbolic tangent function is used in [11] as a smooth transition function. Its general formulation is:

$$
f(t)=c \tanh (a t-b)+d
$$

As explained in [11], tanh varies asymptotically between -1 and 1 so an error $\varepsilon$ should be chosen. For $\varepsilon=10^{-3}$, it can be noticed that $\tanh (3.8) \approx 1-10^{-3}$. For example, for the rising transition $f_{2}$ between $\left[Q_{l o w}, Q_{\text {high }}\right]$ during $\left[T_{f}-T_{\rho}, T_{f}\right]$, the coefficients solving $f_{2}\left(T_{f}-T_{\rho}\right) \approx Q_{\text {low }}$ and $f_{2}\left(T_{f}\right) \approx Q_{\text {high }}$ are:

$$
\begin{aligned}
c & =\frac{Q_{\text {high }}-Q_{\text {low }}}{2} \\
d & =\frac{Q_{\text {high }}+Q_{\text {low }}}{2} \\
a & =\frac{2 \times 3.8}{T_{\rho}} \\
b & =3.8+\frac{2 \times 3.8}{T_{\rho}}\left(T_{f}-T_{\rho}\right)
\end{aligned}
$$

By symmetry, the falling transition $f_{1}$ can be defined similarly. Both functions are summed up:

$f_{1}(t)=\frac{Q_{\text {high }}-Q_{\text {low }}}{2} \tanh \left(3.8-\frac{2 \times 3.8}{T_{2}-T_{1}}\left(t-T_{1}\right)\right)+\frac{Q_{\text {high }}+Q_{\text {low }}}{2}$
$f_{2}(t)=\frac{Q_{\text {high }}-Q_{\text {low }}}{2} \tanh \left(\frac{2 \times 3.8}{T_{4}-T_{3}}\left(t-T_{3}\right)-3.8\right)+\frac{Q_{\text {high }}+Q_{\text {low }}}{2}$

with $T_{1}=0, T_{2}=T_{\rho}, T_{3}=T_{f}-T_{\rho}, T_{4}=T_{f}$. However, those functions can be generalized, independently from the value of the error:

$f_{1}(t)=\frac{Q_{\text {high }}-Q_{\text {low }}}{2} \tanh \left(\mathcal{K}_{1}-\frac{\mathcal{K}_{1}+\mathcal{K}_{2}}{T_{2}-T_{1}}\left(t-T_{1}\right)\right)+\frac{Q_{\text {high }}+Q_{\text {low }}}{2}$
$f_{2}(t)=\frac{Q_{\text {high }}-Q_{\text {low }}}{2} \tanh \left(\frac{\mathcal{K}_{1}+\mathcal{K}_{2}}{T_{4}-T_{3}}\left(t-T_{3}\right)-\mathcal{K}_{2}\right)+\frac{Q_{\text {high }}+Q_{\text {low }}}{2}$

It is then possible to change the shape of the transition curve. $\mathcal{K}_{1}$ designs the error at the moment of the curve where it should converge to $Q_{h i g h}$. In order to have a more smooth motion, the value of $\mathcal{K}_{2}$ should be increased, reducing the error $\varepsilon$ that makes the convergence period to $Q_{\text {low }}$ to last longer. Fig. 2 illustrates this concept with $\left\{\mathcal{K}_{1}, \mathcal{K}_{2}\right\}=\{3.8,3.8\}$ and $\left\{\mathcal{K}_{1}, \mathcal{K}_{2}\right\}=\{3.8,16\}$.

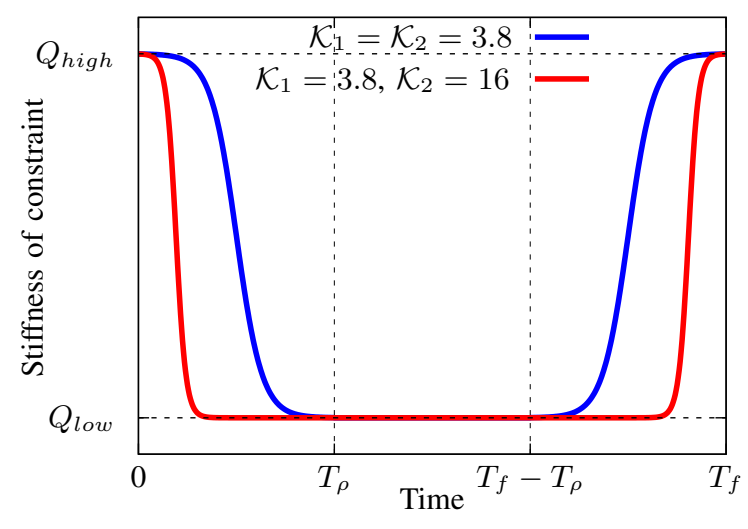

Fig. 2. Hyperbolic tangent transition profile

2) Derivative Hyperbolic Tangent Transition: Inspired by $1-\tanh ^{2}$, which is the derivative function of tanh, we propose the following transition function:

$$
f(t)=-c \tanh ^{2}(a t-b)+d
$$

As $\tanh ^{2}(0)=0$ and $\lim _{t \rightarrow \pm \infty} \tanh ^{2}(t)=1$, we also need to define, as before, an error $\varepsilon$. With $\mathcal{K}^{\prime}>0$ such that $\tanh ^{2}\left(\mathcal{K}^{\prime}\right)=1-\varepsilon$ and $T_{1}=0, T_{2}=T_{\rho}, T_{3}=T_{f}-T_{\rho}$, $T_{4}=T_{f}$, we define the two transition functions:

$$
\begin{aligned}
& f_{1}(t)=-\left(Q_{\text {high }}-Q_{\text {low }}\right) \tanh ^{2}\left(\frac{\mathcal{K}^{\prime}}{T_{2}-T_{1}}\left(t-T_{1}\right)\right)+Q_{\text {high }} \\
& f_{2}(t)=-\left(Q_{\text {high }}-Q_{\text {low }}\right) \tanh ^{2}\left(\frac{\mathcal{K}^{\prime}}{T_{4}-T_{3}}\left(t-T_{3}\right)-\mathcal{K}^{\prime}\right)+Q_{\text {high }}
\end{aligned}
$$

The value of $\mathcal{K}^{\prime}$ will be further discussed in Section IV, however, Fig. 3 gives an example showing the impact of $\mathcal{K}^{\prime}$.

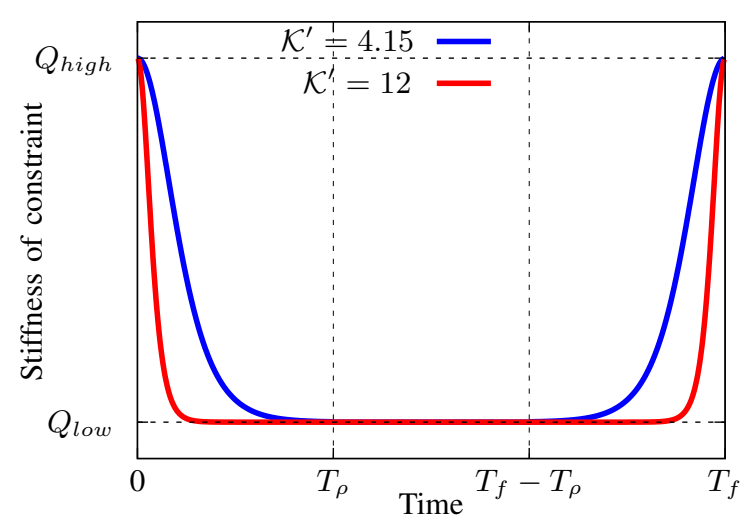

Fig. 3. Derivative hyperbolic tangent transition profile

3) $5^{\text {th }}$ order Transition: In [10], the transition is done using two 5 degrees functions $f_{1}$ and $f_{2}$ with the following limit conditions, with the first and second derivatives equal 
to zero at the beginning and the end of the transitions:

$$
\begin{aligned}
& f_{1}(0)=f_{2}\left(T_{f}\right)=Q_{\text {high }} \\
& f_{1}\left(T_{\rho}\right)=f_{2}\left(T_{f}-T_{\rho}\right)=Q_{\text {low }} \\
& \dot{f}_{1}(0)=\dot{f}_{1}\left(T_{\rho}\right)=\dot{f}_{2}\left(T_{f}\right)=\dot{f}_{2}\left(T_{f}-T_{\rho}\right)=0 \\
& \ddot{f}_{1}(0)=\ddot{f}_{1}\left(T_{\rho}\right)=\ddot{f}_{2}\left(T_{f}\right)=\ddot{f}_{2}\left(T_{f}-T_{\rho}\right)=0
\end{aligned}
$$

With these constraints, the coefficients of the polynomial functions can be uniquely determined. Fig. 1 has been produced using this formulation.

4) Combined Functions Transition: We propose here a new formulation ensuring a better smoothness using two functions. The first of $4^{\text {th }}$ order, $f_{1}$, and the second of $3^{\text {rd }}$ order, $g_{1}$, to reach an intermediate value $Q_{i}$ at $t=T_{i}$ :

- boundary conditions:

$$
\begin{aligned}
f_{1}(0) & =Q_{\text {high }} \\
\dot{f}_{1}(0) & =0 \\
g_{1}\left(T_{\rho}\right) & =Q_{\text {low }} \\
\dot{g}_{1}\left(T_{\rho}\right) & =\ddot{g}_{1}\left(T_{\rho}\right)=0
\end{aligned}
$$

- continuity conditions:

$$
\begin{aligned}
& g_{1}\left(T_{i}\right)=Q_{i} \\
& f_{1}\left(T_{i}\right)=Q_{i} \\
& \dot{f}_{1}\left(T_{i}\right)=\dot{g}_{1}\left(T_{i}\right) \\
& \ddot{f}_{1}\left(T_{i}\right)=\ddot{g}_{1}\left(T_{i}\right)
\end{aligned}
$$

Then, using $f_{2}$ and $g_{2}$ symmetrical to $f_{1}$ and $g_{1}$ in order to get a profile similar to $4, f_{e}$ is defined by:

$$
f_{e}(t)= \begin{cases}f_{1}(t) & \text { if } 0 \leq t \leq T_{i} \\ g_{1}(t) & \text { if } T_{i} \leq t \leq T_{\rho} \\ Q_{\text {low }} & \text { if } T_{\rho} \leq t \leq T_{f}-T_{\rho} \\ g_{2}(t) & \text { if } T_{f}-T_{\rho} \leq t \leq T_{f}-T_{i} \\ f_{2}(t) & \text { otherwise }\end{cases}
$$

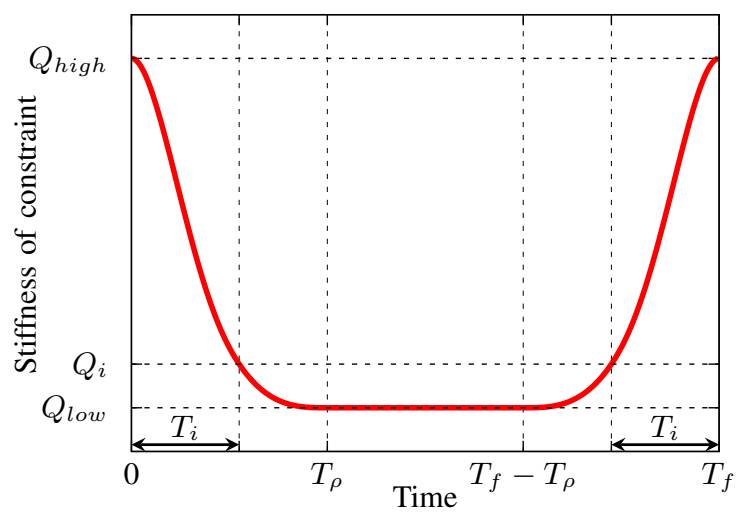

Fig. 4. Combined functions transition profile

\section{RESUlTS}

To achieve the trajectory relaxation, different formulations have been proposed with variations in the objective function and the trajectory constraint in Section III-A and the transition profiles have been discussed in Section IIIB. In this section, the above mentioned methods are tested in simulation to: I)- compare the different QP formulations and eventually recommend one, II)- study the impact of the transitions functions on the Cartesian trajectory of the endeffector of the robot.

The requirements are particularly that the trajectory should deviate continuously from the starting pose, the robot should reach the goal state and the movement is smooth enough, especially when rejoining the reference trajectory, meaning that the movement should not be too fast in the Cartesian space.

The experiments involve the execution of a trajectory, which is mainly parallel to the vertical axis ( $z$ axis), at the same time we consider maximizing the objective function of manipulability index. As will be seen thereafter, the latter objective leads the robot to a configuration, around $Z=0$, that has a good manipulability index. The main role of the transition functions is to make the robot leave this position and go back to the reference trajectory.

Tests have been conducted in simulation with the Baxter robot from Rethink Robotics and the QP problem has be solved by qpOASES [9]. A validation with the real robot has also been carried out as shown in Fig. 5.

\section{A. Formulation of the relaxation}

First, we examined the trajectory constraint, it can be an equality constraint, as in (6), or an inequality constraint as (7). As the inequality is a softer constraint rather than an equality, it should give more liberty for the solver. However, in practice, we have not been able to see any difference with our simple scenario, as (6) and (7) gave the same deviation and Cartesian trajectory.

Then the form of the objective function is studied. The goal is to verify that our method is able to make $\delta$ converge to zero at the end and the beginning. We used the method as described in [10], (6), and compare it to adding the linear part, $\boldsymbol{\xi}^{T} \boldsymbol{\delta}$, as in (8). When using the quadratic form, (6), with $Q_{\text {hard }}=10^{6}$ the components of $\delta$ converge to $10^{-4}$. With $Q_{\text {hard }}=10^{4}$ the components of $\delta$ converge to $10^{-2}$. Thus, decreasing $Q_{h i g h}$ soften the constraint of $\delta$ to converge to zero. However, when adding the linear component to the objective function, (8), with $Q_{\text {hard }}=10^{4}$, we have $\delta$ converging to $10^{-17}$.

As a result, if $Q_{h i g h}$ has to be decreased, adding a linear component to the objective function helps forcing the convergence to zero. That situation can be encountered when the QP problem has to solve several objectives, they often interfere on each others and each associated $\alpha$ or weighting matrix $Q$ has to be set to reflect the compromise between the objectives. In that context, some coefficients might need to be decreased. Adding then a linear component to the relaxation 
seems to be an interesting solution to ensure the convergence to the reference trajectory.

\section{B. Comparing the transition functions}

In this section, we compare the modified Cartesian trajectory generated by our algorithm with the transition functions regarding the objective of having a smooth behavior. The parameters used in the experiments are: $Q_{\text {hard }}=10^{6}, Q_{\text {soft }}=1, T_{\rho}=0.2 \times T_{f}$.

In Fig. 6, the $5^{\text {th }}$ order function was used. It shows that the effective transition in the Cartesian space is a lot faster than the transition that can be seen in Fig. 1 which lasts for $T_{\rho}$ seconds. It means that when the value of $Q_{\delta}$ becomes slightly greater than $Q_{\text {low }}$, even a small variation is sufficient to make the arm move fast to rejoin the reference trajectory. However, it is mandatory to have a high $Q_{h i g h}$ in order to have a negligible error at the end (the same remarks apply by symmetry at the beginning of the task when converging to $Q_{\text {low }}$ ).

Then, $Q_{\delta}$ should vary very slowly and with low amplitude when increasing from $Q_{\text {low }}$ or converging to it, so that the velocity of the arm in the Cartesian space is acceptable. Furthermore, $Q_{\delta}$ should vary indifferently to or from $Q_{h i g h}$.

When observing Fig. 2, the hyperbolic tangent function seems to satisfy the above requirements. In particular, increasing $\mathcal{K}_{2}$ leads to a very slow transition to or from $Q_{\text {low }}$. However, as shown in Fig. 7, even though increasing $\mathcal{K}_{2}$ have indeed made the movement a little more smooth during the transition, the period of transition has approximatively the same duration. Thus, the movement still appear very fast in the Cartesian space.

Moreover, the derivative hyperbolic tangent function that seems in Fig. 3 to have an even longer transition period, it does not have a significant impact on the results, even though the transition movement with $\mathcal{K}^{\prime}=12$ seems to produce the longer transition period and smoother movement so far as can be seen in Fig. 8. The above observations are more clear in the velocity and acceleration domains as shown in Fig. 10 and Fig. 11.

The main reason of the above-mentioned phenomena is that we have very low control on the shape of the functions. In particular, the variable $\mathcal{K}_{2}$ and $\mathcal{K}^{\prime}$ that we used as control variables represent the errors at the limits of the transition period, increasing them means the error is decreased and it deforms the curve of the function, thus leading to a longer transition. However, the amplitude of $Q_{\delta}$ is not easily controlled, which is behind the fast movements.

The solution is brought by the combined functions method because the intermediate value and time is directly controllable. Fig. 9 shows the results with the intermediate variables: $Q_{i}=100, T_{i}=0.3 \times T_{\rho}$. As a result, the transition is efficiently controlled and has a smooth transition producing a satisfying Cartesian movement.

We have also validated the method with the combined functions on Baxter research robot as shown in Fig. 5.

\section{CONCLUSION}

In this paper, we presented the formulation of trajectory relaxation, which can be seen as an improvement to the classical Inverse Kinematics (IK) problem formulation. In addition to the already known definitions, we have also proposed several variations as potential solutions to increase the robustness of the resulting optimization problem.

Moreover, the transition functions between the hard constraint state of following the reference trajectory and the relaxed state have been extensively studied, we have mainly proposed new formulations and some insights into improving some existing functions and compared their impact on the Cartesian trajectory, especially regarding the resulting velocity of the movement. The results revealed that the approach of combining two functions that are connected at an intermediate value gave good results to control and produce a smooth and slow transition in the Cartesian space.

\section{ACKNOWLEDGMENT}

This research is supported by the Natural Sciences and Engineering Research Council of Canada (NSERC) under the grant RGPIN-2012-419406.

\section{REFERENCES}

[1] K. Dufour and W. Suleiman, "On integrating manipulability index into inverse kinematics solver," in IEEE/RSJ International Conference on Intelligent Robots and Systems (IROS), 2017, pp. 6967-6972.

[2] F. Kanehiro, F. Lamiraux, O. Kanoun, E. Yoshida, and J.-P. Laumond, "A Local Collision Avoidance Method for Non-strictly Convex Objects," in 2008 Robotics: Science and Systems Conference, Zurich, Switzerland, June 2008.

[3] F. Kanehiro, M. Morisawa, W. Suleiman, K. Kaneko, and E. Yoshida, "Integrating geometric constraints into reactive leg motion generation," in IEEE/RSJ International Conference on Intelligent Robots and Systems (IROS), 2010, pp. 4069-4076.

[4] O. Kanoun, "Real-time prioritized kinematic control under inequality constraints for redundant manipulators," in Proceedings of Robotics: Science and Systems, Los Angeles, CA, USA, June 2011.

[5] A. Escande, N. Mansard, and P.-B. Wieber, "Fast resolution of hierarchized inverse kinematics with inequality constraints," in IEEE International Conference on Robotics and Automation (ICRA). IEEE, 2010, pp. 3733-3738.

[6] _ - "Hierarchical quadratic programming: Fast online humanoidrobot motion generation," The International Journal of Robotics Research, vol. 33, pp. 1006-1028, 2014.

[7] W. Suleiman, "On inverse kinematics with inequality constraints: new insights into minimum jerk trajectory generation," Advanced Robotics, vol. 30, no. 17-18, pp. 1164-1172, 2016.

[8] T. Yoshikawa, "Manipulability of Robotic Mechanisms," The International Journal of Robotics Research, vol. 4, no. 2, pp. 3-9, 1985.

[9] H. Ferreau, C. Kirches, A. Potschka, H. Bock, and M. Diehl, "qpOASES: A parametric active-set algorithm for quadratic programming," Mathematical Programming Computation, vol. 6, no. 4, pp. 327-363, 2014.

[10] F. Kanehiro, W. Suleiman, K. Miura, M. Morisawa, and E. Yoshida, "Feasible pattern generation method for humanoid robots," IEEERAS 9th International Conference on Humanoid Robots, pp. 542-548, 2009.

[11] K. Cole and A. M. Wickenheiser, "Reactive trajectory generation in an unknown environment," in IEEE/RSJ International Conference on Intelligent Robots and Systems (IROS), 2017, pp. 2151-2157. 

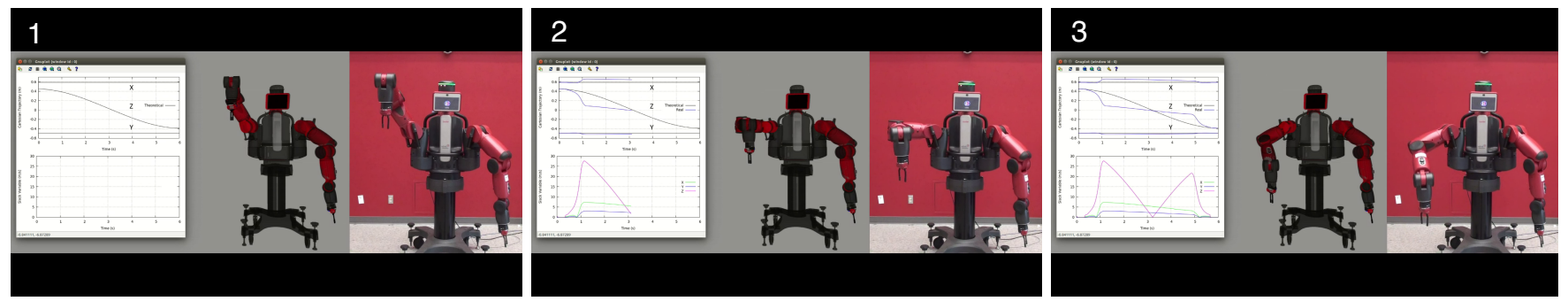

Fig. 5. Snapshots of the validation of the method with combined functions of $4^{\text {th }}$ and $3^{\text {rd }}$ orders (Section III-B.4) on the Baxter research robot.

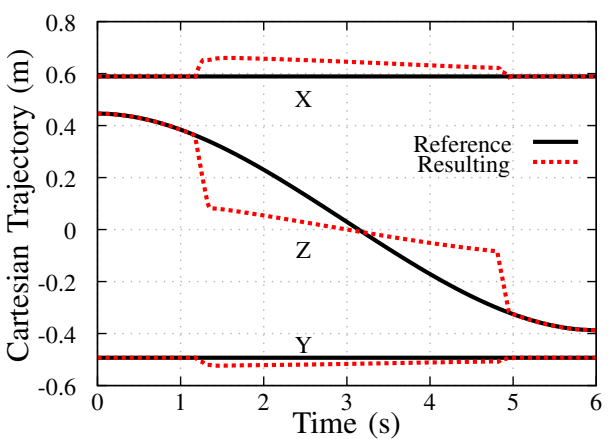

Fig. 6. Reference and computed trajectories for $5^{\text {th }}$ order function transition

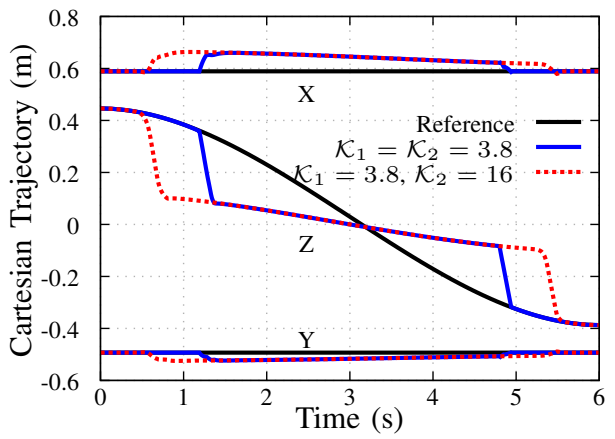

Fig. 7. Reference and computed trajectories for hyperbolic tangent transition

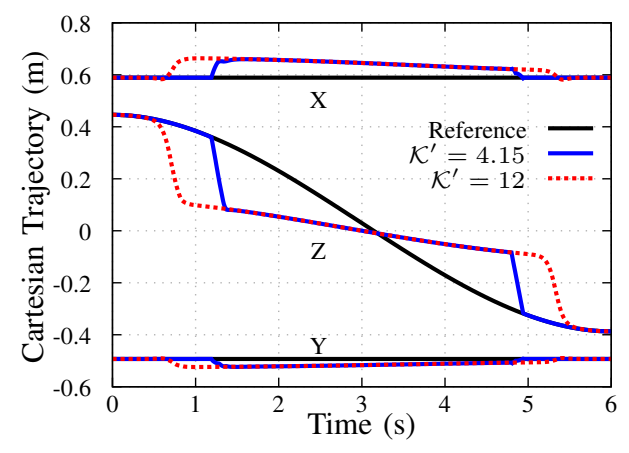

Fig. 8. Reference and computed trajectories for derivative hyperbolic tangent transition

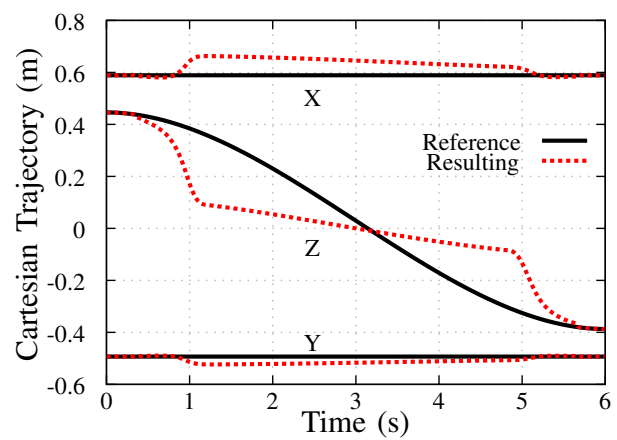

Fig. 9. Reference and computed trajectories for combined functions transition

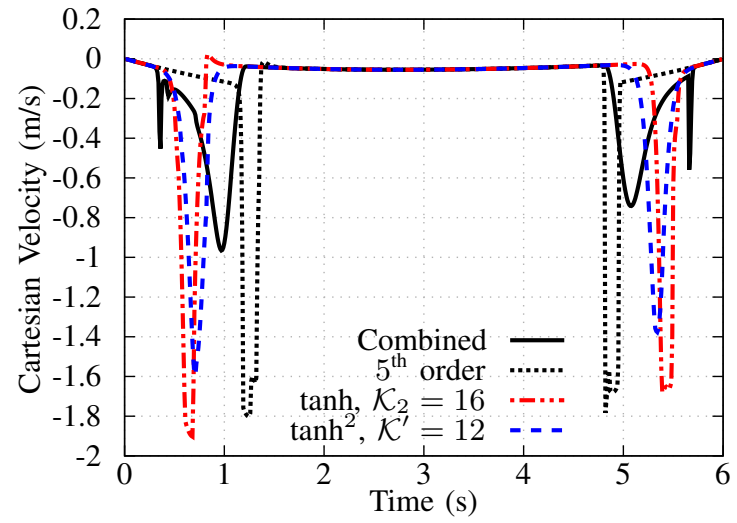

Fig. 10. Resulting velocity profiles of the different transition functions

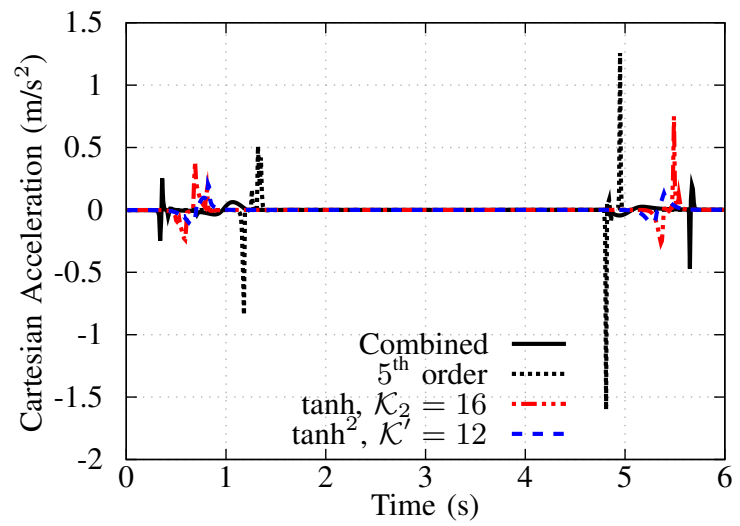

Fig. 11. Resulting acceleration profiles of the different transition functions 